\title{
Correction to "First Trimester Procedural Abortion in Family Medicine"
}

In the abovementioned article (Lyus RJ, Gianutsos P, and Gold M.J Am Board Fam Med 2009;22(2): 169-74), there was an error in the abstract. The abstract should have read that "almost one-third of all women will have at least one abortion during their lifetime." We apologize for the error, and we regret any confusion or inconvenience it may have caused. (doi: 10.3122/jabfm.2009.06.090235.) 